\title{
ESTUDIO BIBLIOMÉTRICO DE LA EVOLUCIÓN DE LA REVISTA ENSEÑANZA DE LAS CIENCIAS A PARTIR DE SUS FUENTES DE INFORMACIÓN
}

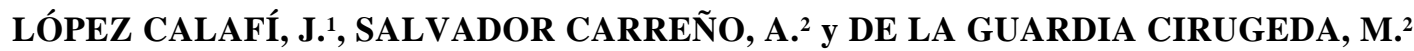 \\ 1 IES Blasco Ibáñez. 46400 Cullera. València. \\ ${ }^{2}$ Departament de Química Analítica. Facultat de Química. Universitat de València.
}

\section{SUMMARY}

Science learning research development in Spain can be bibliometrically analyzed using Enseñanza de las Ciencias as indicator, because of the incidence level of this journal in researchers with interest in scientific education. The references which are cited in the articles have been studied in order to analyze the main sources and the most productive authors, using the Bradford model. The evolution of the type of sources and articles which are cited during the period of time studied are considered.

\section{INTRODUCCIÓN}

Enseñanza de las Ciencias es sin duda, en nuestro país, la revista que recoge la mayor parte de los estudios de investigación en didáctica de las ciencias realizados por grupos españoles, tanto en los niveles de enseñanza media como a nivel universitario. Por ello, el estudio de los trabajos publicados en esta revista puede ser utilizado como un buen indicador del tipo de investigación didáctica que se está llevando a cabo durante los últimos años (Moreira, 1994; Gil, 1994).

En el presente trabajo, se han analizado los artículos publicados en Enseñanza de las Ciencias entre los años 1983 (fecha en que se inició su publicación) y 1996, mediante la aplicación de tratamientos bibliométricos.

El soporte utilizado en este estudio han sido las referencias citadas por los distintos autores en sus artículos, es decir, las fuentes de información de mayor interés para ellos en relación con el tema de sus trabajos.

A lo largo de este estudio, se han analizado distintos aspectos relativos a las referencias de los artículos publi- cados en Enseñanza de las Ciencias en el período considerado.

En primer lugar se ha contabilizado la evolución del número de referencias durante estos años, así como el soporte al que se refieren (libros, artículos, comunicaciones en congresos, etc.), y en particular las citas referidas a otros artículos de la propia Enseñanza de las Ciencias (autocitas).

Para conocer cuáles han sido las fuentes de información que han tenido una mayor influencia sobre las investigaciones en didáctica de las ciencias en España, se ha realizado un estudio bibliométrico, basado en un modelo propuesto por Bradford (1948), que permite identificar el conjunto de referencias más citadas y que se puede considerar como núcleo del total de la población de citas. Esta adaptación del modelo de Bradford ha sido propuesta y utilizada por los autores del presente artículo en otros trabajos anteriores en los que se realizaron estudios sobre algunos temas concretos de la química (López et al., 1985), de la didáctica de la química (López, 1995) 
y sobre una de las revistas españolas más representativas en el campo de la química (López et al., 1986). Así mismo, se ha estudiado la vigencia temporal del núcleo de referencias más citadas.

Paralelamente, se realiza también un estudio bibliométrico de los autores que publicaron en Enseñanza de las Ciencias durante el período considerado, y se relaciona el núcleo de autores encontrado con el de los autores de las referencias más citadas.

\section{TRATAMIENTO BIBLIOMÉTRICO DE LOS DATOS}

Para el análisis de las referencias más citadas y de los autores más productivos en Enseñanza de las Ciencias se ha utilizado una adaptación del modelo bibliométrico de Bradford. (Bradford, 1948; López et al., 1985; López et al., 1986; López, 1995).

El tratamiento original de Bradford consistía en ordenar los autores en orden decreciente de productividad para representar el número acumulado de comunicaciones o artículos presentados, frente al logaritmo del número acumulado de autores.

Para realizar esta representación, previamente se ordenan de mayor a menor los autores según el número de trabajos realizados por cada uno de ellos. Se van sumando progresivamente el número de autores $(x)$ y el número de publicaciones de manera acumulativa $(y)$, y se representa $y=f(\log x)$, de tal forma que un punto cualquiera de la curva se obtiene a partir del número de trabajos totales que han publicado un determinado número de autores.

La representación logarítmica permite la obtención de diferentes pendientes en las que se distribuyen los autores con una productividad análoga. La primera zona de la curva se denomina núcleo e incluye a los autores más productivos.

Este tratamiento se puede adaptar para identificar revistas con mayor productividad en un tema determinado o, como se ha hecho en este caso, las referencias con un mayor número de citas.

\section{ESTUDIO DE LAS REFERENCIAS CITADAS EN LOS ARTÍCULOS DE ENSENANZA DE LAS CIENCIAS}

\section{Evolución del número de refencias citadas en los trabajos}

Se han contabilizado los trabajos publicados en la revista y el número de referencias citadas en ellos en función del año de su publicación, obteniéndose los datos de la tabla I.
Tabla I

Estudio de los trabajos publicados.

\begin{tabular}{|c|c|c|c|}
\hline Año & $\begin{array}{c}\text { Número } \\
\text { de trabajos }\end{array}$ & $\begin{array}{c}\text { Número } \\
\text { de referencias }\end{array}$ & $\begin{array}{c}\text { Referencias } \\
\text { Trabajos }\end{array}$ \\
\hline 1983 & 28 & 267 & 10 \\
1984 & 25 & 298 & 12 \\
1985 & 27 & 229 & 8 \\
1986 & 23 & 678 & 29 \\
1987 & 28 & 470 & 17 \\
1988 & 28 & 653 & 23 \\
1989 & 30 & 715 & 24 \\
1990 & 32 & 651 & 20 \\
1991 & 31 & 772 & 25 \\
1992 & 37 & 807 & 22 \\
1993 & 35 & 978 & 28 \\
1994 & 39 & 1.241 & 32 \\
1995 & 32 & 847 & 26 \\
1996 & 33 & 1.270 & 38 \\
\hline TOTAL & 428 & 9.874 & 23 \\
\hline
\end{tabular}

Se puede observar que tanto el número de trabajos como el número de referencias citadas por trabajo han ido aumentando a lo largo de los años, estabilizándose alrededor de los 30-35 trabajos publicados por año, y con una media de aproximadamente 30 citas por trabajo, lo cual constituye el perfil medio de las publicaciones de Enseñanza de las Ciencias.

\section{Soporte en el que se han publicado las referencias}

Las referencias citadas en los artículos de la revista se han clasificado año por año atendiendo a su soporte, es decir, si el trabajo de referencia es otro artículo de revista, un libro u otro material, como comunicaciones en congresos, tesis, etc. En la figura 1 se observa cómo, de un total de 9.874 referencias citadas en el período 1983-96, el 50,57\% son artículos de revistas, el 39,16\% se refiere a libros, mientras que el $10,27 \%$ corresponde a otro tipo de soporte.

Al mismo tiempo, se contabilizaron las autocitas de la revista (citas de artículos anteriores de Enseñanza de las Ciencias) con el fin de constatar la importancia que iba adquiriendo la revista en la literatura posterior.

Los resultados obtenidos se muestran en la tabla II. En la figura 2a podemos observar cómo el porcentaje de libros citados sufre una ligera disminución a lo largo de los años, incrementándose al mismo tiempo el de los artículos de revistas, lo que da una idea de la maduración de la investigación española en didáctica de las ciencias. 
Figura 1

Soporte de las referencias citadas en la revista Enseñanza de la Ciencias durante el período 1983-96.

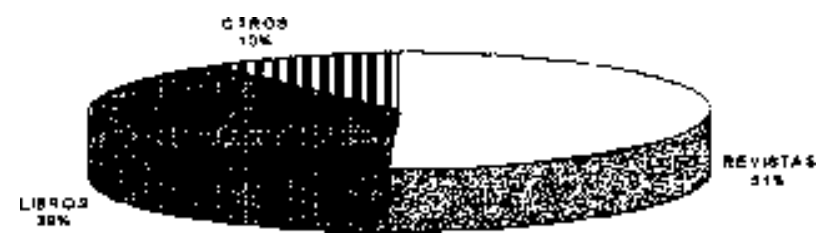

Del mismo modo se puede observar en la figura $2 \mathrm{~b}$ el incremento del porcentaje de autocitas de la revista, lo que evidencia un aumento del «prestigio» de la misma en la comunidad didáctico-científica nacional, su implantación como fuente de orientación de la investigación en este campo y la continuidad de las escuelas y grupos de investigación que publican en la revista.

\section{ESTUDIO DE LAS REFERENCIAS MÁS CITADAS}

\section{Obtención bibliométrica del núcleo principal}

Las referencias citadas por todos los autores que han publicado trabajos en la revista Enseñanza de las Ciencias se han ordenado atendiendo al número de veces que se repetían, y en el anexo I se muestran las referencias más citadas en Enseñanza de las Ciencias a lo largo de todo el período considerado.

Cabe destacar el libro de Ausubel y otros, la referencia más citada, en un $17,5 \%$ de los trabajos, tanto en su edición original como en su traducción castellana, que junto a las de Posner y otros (citado en el 15\%) y Driver (citado en el 11\%), evidencian la tendencia de los autores a citar trabajos relacionados con el cambio conceptual y los problemas asociados con la psicología cognoscitiva de los alumnos.

Estudio de las referencias citadas en los trabajos publicados en la revista Enseñanza de las Ciencias.

\begin{tabular}{|c|c|c|c|c|c|c|c|}
\hline AÑO & $\begin{array}{c}\text { NÚM. } \\
\text { REFERENCIAS }\end{array}$ & $\begin{array}{c}\text { NÚM. } \\
\text { LIBROS }\end{array}$ & $\begin{array}{c}\% \\
\text { LIBROS }\end{array}$ & $\begin{array}{c}\text { NÚM. } \\
\text { REVISTAS }\end{array}$ & $\begin{array}{c}\% \\
\text { REVISTAS }\end{array}$ & AUTOCITAS & $\begin{array}{c}\% \\
\text { AUTOCITAS }\end{array}$ \\
\hline 1983 & 267 & 140 & 52 & 90 & 34 & 0 & 0 \\
\hline 1984 & 298 & 141 & 47 & 114 & 38 & 9 & 3,0 \\
\hline 1985 & 229 & 116 & 51 & 93 & 41 & 21 & 9,2 \\
\hline 1986 & 678 & 190 & 2 & 356 & 53 & 27 & 4,2 \\
\hline 1987 & 470 & 185 & 39 & 250 & 53 & 30 & 6,4 \\
\hline 1988 & 653 & 297 & 45 & 248 & 38 & 45 & 6,9 \\
\hline 1989 & 715 & 320 & 45 & 315 & 44 & 58 & 8,1 \\
\hline 1990 & 651 & 265 & 41 & 322 & 49 & 42 & 6,5 \\
\hline 1991 & 772 & 296 & 38 & 400 & 52 & 70 & 9,1 \\
\hline 1992 & 807 & 282 & 35 & 449 & 56 & 81 & 10,0 \\
\hline 1993 & 978 & 410 & 42 & 486 & 50 & 111 & 11,3 \\
\hline 1994 & 1.241 & 535 & 43 & 629 & 51 & 112 & 9,0 \\
\hline 1995 & 847 & 275 & 32 & 501 & 59 & 81 & 9,6 \\
\hline 1996 & 1.270 & 415 & 33 & 730 & 57 & 105 & 8,3 \\
\hline TOTAL & 9.874 & 3.867 & 39 & 4.993 & 51 & 792 & 8,0 \\
\hline
\end{tabular}


Figura 2a

Estudio de las referencias citadas en la revista Enseñanza de las Ciencias en función del año de publicación y del soporte.

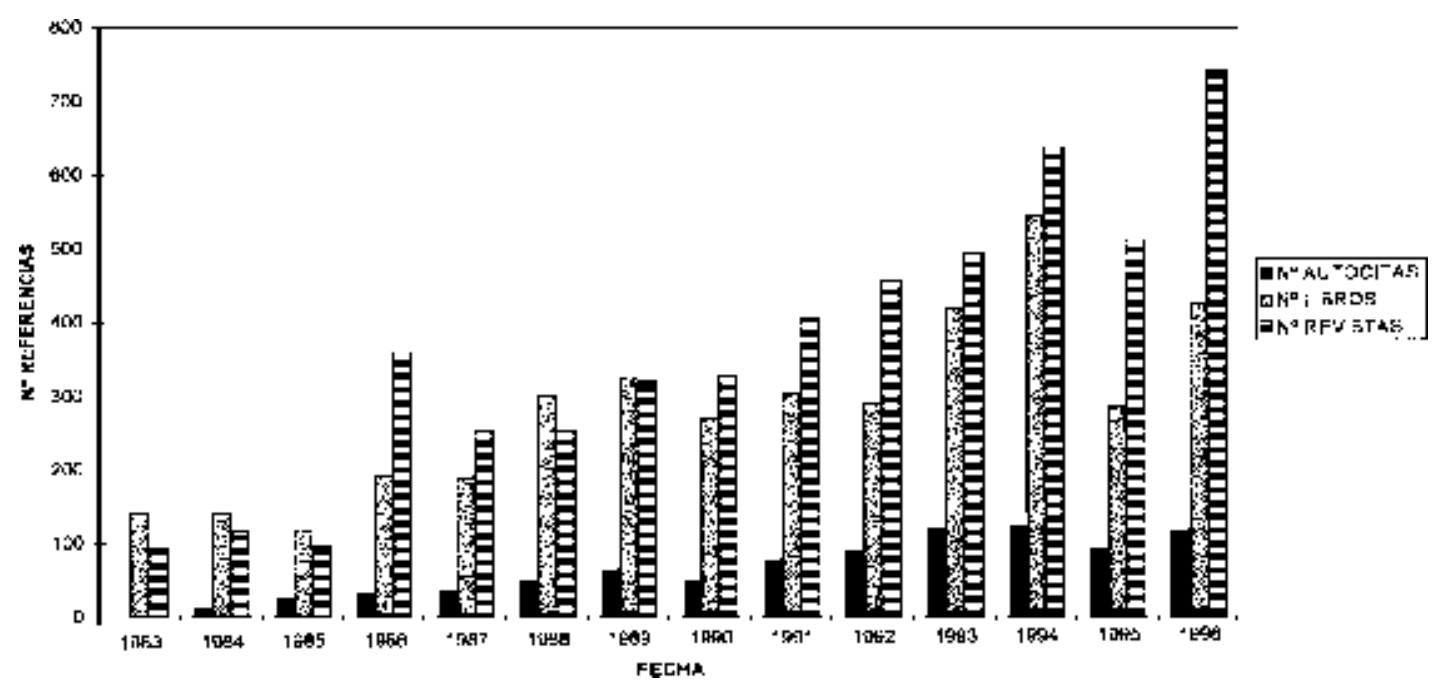

Se ha adaptado el tratamiento bibliométrico de Bradford descrito anteriormente, para conocer en este caso el núcleo de las referencias más citadas, estimado mediante la identificación de la zona lineal de la curva de Bradford. Para ello se consideran los datos de la tabla III.

La curva de Bradford de la figura 3 se ha construido representando el número acumulado de citas recibidas por el total de referencias citadas en los trabajos publicados durante el período considerado, en función del logaritmo del número acumulado de dichas referencias.
Se observan cuatro cambios de pendiente en la figura que responden a cuatro conjuntos de referencias con un número similar de citas recibidas. El núcleo es el formado por las 7 referencias más citadas del anexo I.

Estas 7 referencias acumulan un total de 345 citas, lo que constituye el $3,5 \%$ de todas las citas, habiéndose publicado algunos artículos en la revista Enseñanza de las Ciencias que citan varias e incluso todas estas referencias, lo que da idea de la importancia de las mismas en las líneas de investigación llevada a cabo por los autores.

Figura $2 b$

Referencia cuyo soporte es la propia revista Enseñanza de las Ciencias (autocitas).

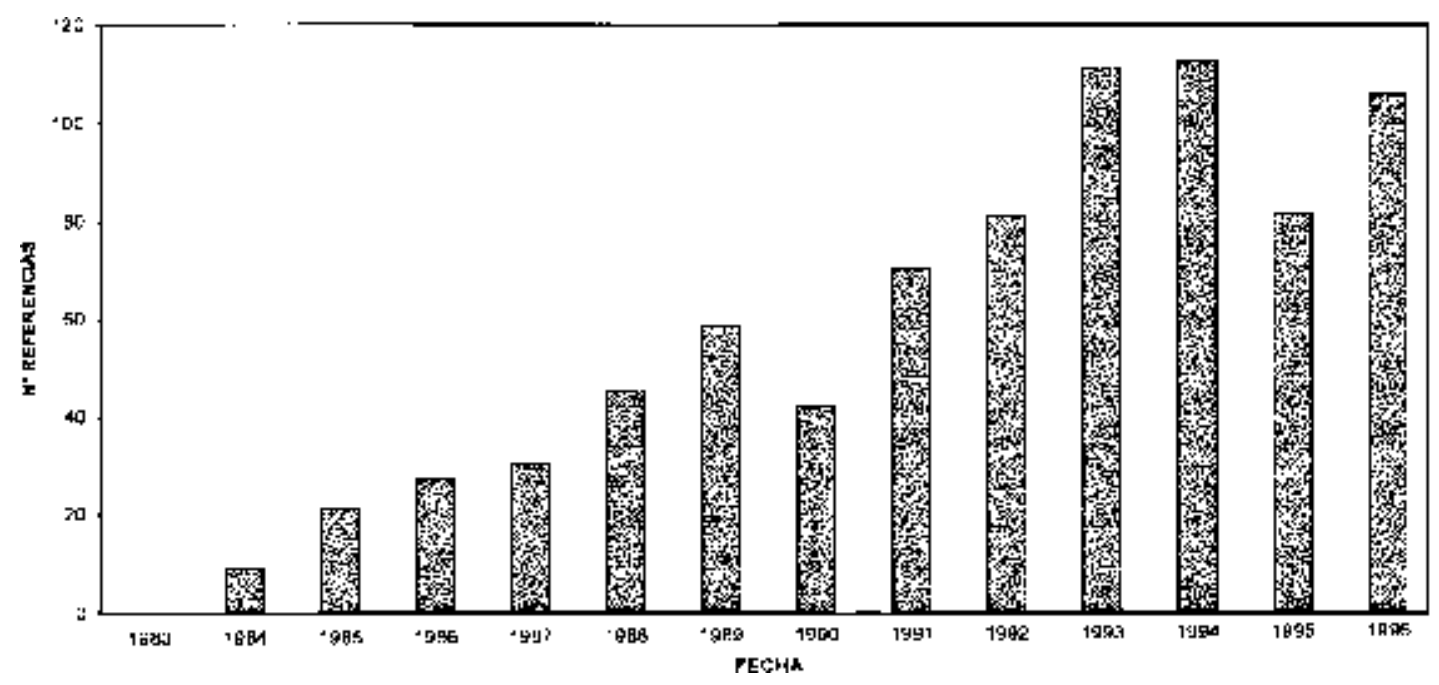




\section{Vigencia de las referencias que constituyen el núcleo}

Se ha estudiado año por año el número de citas recibidas por las siete referencias más citadas con objeto de discernir si su línea de investigación mantiene su vigencia o si, por el contrario, el número de artículos que las cita cada vez es menor, lo que evidenciaría un agotamiento de dicha tendencia y su paulatina sustitución por otras nuevas.

Se han representado las curvas de crecimiento de las citas recibidas por las referencias más citadas. En la tabla IV se indica el número de citas anuales $(\mathrm{Ni})$ y el número acumulado de citas $\left(\sum \mathrm{Ni}\right)$ que reciben estas referencias, y en la figura 4 se muestra la representación del número acumulado de citas recibidas por las siete referencias del núcleo de Bradford.

Podemos observar cómo el libro de Ausubel, un clásico publicado en 1976, presenta un descenso de citas en los últimos años. Lo mismo ocurre en los artículos de Driver (1986) y Gil (1983), que presentan, después de algunos altibajos, un ligero descenso del número de citas.

Por el contrario, los trabajos de Posner y otros de 1982 y Driver y otros de 1985 mantienen ambos todavía plena vigencia, pues sus citas siguen aumentando en 1996.

El libro de Kuhn (1962) es un clásico entre los textos relacionados con la filosofía de la ciencia que todavía perdura a pesar del tiempo que ha pasado desde su publicación. En cuanto al trabajo de Gil-Carrascosa (1985), parece haber llegado a su número máximo de citas anuales, ya que durante los últimos años se observa una estabilización.

\section{ESTUDIO DE LQS AUTORES QUE PUBLI- CAN EN ENSENANZA DE LAS CIENCIAS Y SU RELACION CON LOS TRABAJOS MÁS CITADOS}

\section{Obtención bibliométrica del núcleo principal}

Se ha realizado el estudio de los autores que firman los trabajos publicados en la revista Enseñanza de las Ciencias durante este período, ordenándolos según el número de trabajos publicados (Anexo II).

Para conocer el núcleo de autores más productivos se ha adaptado el tratamiento bibliométrico propuesto por Bradford (1948), empleando los datos de la tabla V.

La curva de Bradford de la figura 5 se ha realizado representando el número acumulado de trabajos publicados por los autores durante el período considerado en función del logaritmo del número acumulado de autores.

Este núcleo está formado por los seis autores indicados en la tabla VI, algunos de los cuales forman parte del profesorado de la Escuela Universitaria de Formación del Profesorado de la Universitat de València.
Tabla III

Relación entre las referencias más citadas en Enseñanza de las Ciencias y el número de citas recibidas.

\begin{tabular}{|c|c|c|c|c|}
\hline $\begin{array}{l}\text { Núm. } \\
\text { referencias }\end{array}$ & $\begin{array}{l}\text { Núm. } \\
\text { citas }\end{array}$ & $\begin{array}{l}\text { Referencias } \\
\text { acumuladas }\end{array}$ & $\begin{array}{c}\text { Citas } \\
\text { acumuladas }\end{array}$ & $\begin{array}{l}\text { Log. } \\
\text { referencias } \\
\text { acumuladas }\end{array}$ \\
\hline 1 & 75 & 1 & 75 & 0 \\
\hline 1 & 64 & 2 & 139 & 0,30 \\
\hline 1 & 47 & 3 & 186 & 0,48 \\
\hline 1 & 46 & 4 & 232 & 0,60 \\
\hline 1 & 44 & 5 & 276 & 0,70 \\
\hline 1 & 35 & 6 & 311 & 0,78 \\
\hline 1 & 34 & 7 & 345 & 0,85 \\
\hline 2 & 31 & 9 & 407 & 0,95 \\
\hline 2 & 27 & 11 & 461 & 1,04 \\
\hline 1 & 26 & 12 & 487 & 1,08 \\
\hline 2 & 24 & 14 & 535 & 1,15 \\
\hline 3 & 23 & 17 & 604 & 1,23 \\
\hline 1 & 22 & 18 & 626 & 1,26 \\
\hline 2 & 21 & 20 & 668 & 1,30 \\
\hline 5 & 20 & 25 & 768 & 1,40 \\
\hline 2 & 19 & 27 & 806 & 1,43 \\
\hline 2 & 18 & 29 & 842 & 1,46 \\
\hline 1 & 17 & 30 & 859 & 1,48 \\
\hline 2 & 16 & 32 & 891 & 1,51 \\
\hline 1 & 15 & 33 & 906 & 1,52 \\
\hline 4 & 14 & 37 & 962 & 1,57 \\
\hline 3 & 13 & 40 & 1.001 & 1,60 \\
\hline 2 & 12 & 42 & 1.025 & 1,62 \\
\hline 5 & 11 & 47 & 1.080 & 1,67 \\
\hline 9 & 10 & 56 & 1.170 & 1,75 \\
\hline 9 & 9 & 65 & 1.251 & 1,81 \\
\hline 11 & 8 & 76 & 1.339 & 1,88 \\
\hline 31 & 7 & 107 & 1.556 & 2,03 \\
\hline 29 & 6 & 136 & 1.730 & 2,13 \\
\hline 42 & 5 & 178 & 1.940 & 2,25 \\
\hline 56 & 4 & 234 & 2.164 & 2,37 \\
\hline 152 & 3 & 386 & 2.620 & 2,59 \\
\hline 445 & 2 & 831 & 3.510 & 2,92 \\
\hline
\end{tabular}

Relación de los autores más productivos con los trabajos más citados

Para establecer la relación entre los autores más productivos y los trabajos más citados, se ha contabilizado el número de veces que estos autores citaban dichos trabajos. Los resultados se pueden observar en la tabla VI.

Se observa que en algún caso existe un número significativo de citas de trabajos propios. También sorprende ver cómo el libro de Driver y otros (1985) sólo es citado en cinco ocasiones y por sólo tres de los autores más productivos, cuando se ha manifestado como una de las obras que gozan de una mayor vigencia, según se ha 
Figura 3

Curva de Bradford de las referencias citadas en los artículos de la revista Enseñanza de las Ciencias.

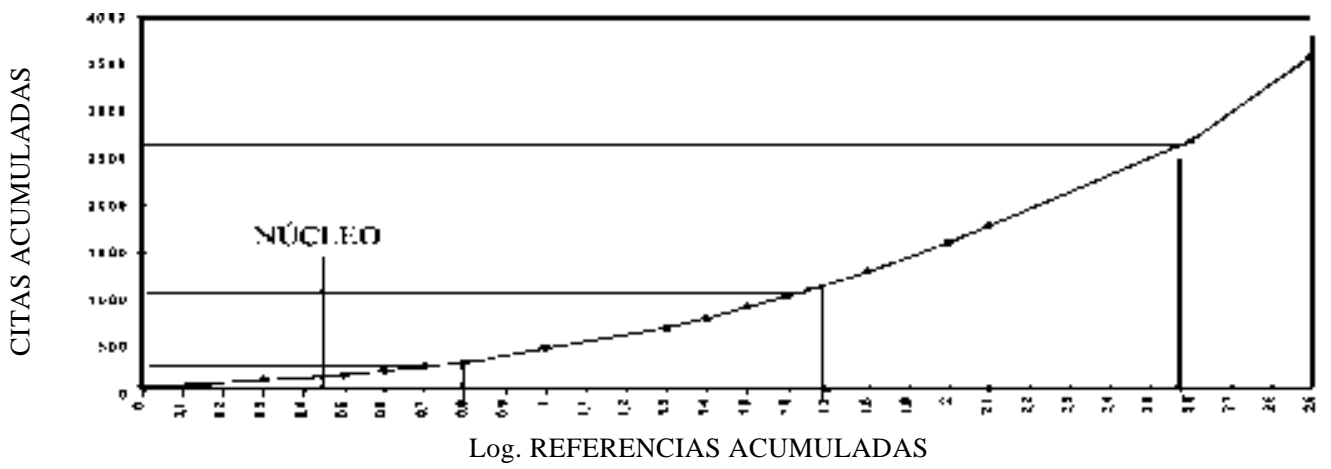

Tabla IV

Evolución de las citas recibidas por las referencias más citadas.

\begin{tabular}{|c|c|c|c|c|c|c|c|c|c|c|c|c|c|c|}
\hline \multirow[t]{2}{*}{ FECHA } & \multicolumn{2}{|c|}{$\begin{array}{c}\text { AUSUBEL } \\
\text { (1976) }\end{array}$} & \multicolumn{2}{|c|}{$\begin{array}{l}\text { POSNER } \\
\text { ET AL. } \\
\text { (1982) }\end{array}$} & \multicolumn{2}{|c|}{$\begin{array}{l}\text { DRIVER } \\
\text { (1986) }\end{array}$} & \multicolumn{2}{|c|}{$\begin{array}{l}\text { KUHN } \\
\text { (1962) }\end{array}$} & \multicolumn{2}{|c|}{$\begin{array}{l}\text { GIL } \\
\text { (1983) }\end{array}$} & \multicolumn{2}{|c|}{$\begin{array}{c}\text { DRIVER } \\
\text { ET AL. } \\
\text { (1985) }\end{array}$} & \multicolumn{2}{|c|}{$\begin{array}{c}\text { GIL- } \\
\text { CARRASCOSA } \\
\text { (1985) }\end{array}$} \\
\hline & $\mathrm{Ni}$ & $\sum \mathrm{Ni}$ & $\mathrm{Ni}$ & $\sum \mathrm{Ni}$ & $\mathrm{Ni}$ & $\sum \mathrm{Ni}$ & $\mathrm{Ni}$ & $\sum \mathrm{Ni}$ & $\mathrm{Ni}$ & $\sum \mathrm{Ni}$ & $\mathrm{Ni}$ & $\sum \mathrm{Ni}$ & $\mathrm{Ni}$ & $\sum \mathrm{Ni}$ \\
\hline 1983 & 4 & 4 & 0 & 0 & 0 & 0 & 4 & 4 & 0 & 0 & 0 & 0 & 0 & 0 \\
\hline 1984 & 2 & 6 & 3 & 3 & 0 & 0 & 2 & 6 & 3 & 3 & 0 & 0 & 0 & 0 \\
\hline 1985 & 1 & 7 & 1 & 4 & 0 & 0 & 2 & 8 & 5 & 8 & 0 & 0 & 1 & 1 \\
\hline 1986 & 2 & 9 & 2 & 6 & 1 & 1 & 3 & 11 & 2 & 10 & 1 & 1 & 1 & 2 \\
\hline 1987 & 7 & 16 & 3 & 9 & 6 & 7 & 3 & 14 & 3 & 13 & 1 & 2 & 2 & 4 \\
\hline 1988 & 9 & 25 & 7 & 16 & 7 & 14 & 7 & 21 & 5 & 18 & 5 & 7 & 4 & 8 \\
\hline 1989 & 4 & 29 & 5 & 21 & 7 & 21 & 3 & 24 & 6 & 24 & 1 & 8 & 3 & 11 \\
\hline 1990 & 7 & 36 & 7 & 28 & 3 & 24 & 3 & 27 & 4 & 26 & 3 & 11 & 1 & 12 \\
\hline 1991 & 12 & 48 & 6 & 32 & 5 & 29 & 1 & 28 & 5 & 31 & 3 & 14 & 5 & 17 \\
\hline 1992 & 7 & 55 & 4 & 36 & 4 & 33 & 4 & 32 & 5 & 36 & 1 & 15 & 5 & 22 \\
\hline 1993 & 9 & 64 & 7 & 43 & 6 & 39 & 3 & 35 & 4 & 40 & 5 & 20 & 2 & 24 \\
\hline 1994 & 4 & 68 & 5 & 48 & 2 & 41 & 4 & 39 & 2 & 42 & 4 & 24 & 3 & 27 \\
\hline 1995 & 2 & 70 & 7 & 55 & 3 & 44 & 2 & 41 & 0 & 42 & 4 & 28 & 2 & 29 \\
\hline 1996 & 6 & 76 & 9 & 64 & 3 & 47 & 5 & 46 & 2 & 44 & 7 & 35 & 5 & 34 \\
\hline
\end{tabular}


Tabla V

Relación entre los autores que publican en Enseñanza de las Ciencias y el número de trabajos que han publicado en el período 1983-96.

\begin{tabular}{|c|c|c|c|c|}
\hline $\begin{array}{c}\text { Núm. } \\
\text { autores }\end{array}$ & $\begin{array}{c}\text { Núm. } \\
\text { autores }\end{array}$ & $\begin{array}{c}\text { Autores } \\
\text { acumulados }\end{array}$ & $\begin{array}{c}\text { Trabajos } \\
\text { acumulados }\end{array}$ & $\begin{array}{c}\text { Log. autores } \\
\text { acumulados }\end{array}$ \\
\hline 1 & 10 & 1 & 10 & 0 \\
\hline 1 & 8 & 2 & 18 & 0,30 \\
\hline 2 & 7 & 4 & 32 & 0,60 \\
\hline 2 & 6 & 6 & 44 & 0,78 \\
\hline 5 & 5 & 11 & 69 & 1,04 \\
\hline 10 & 4 & 21 & 109 & 1,32 \\
\hline 28 & 3 & 49 & 193 & 1,69 \\
\hline 98 & 2 & 147 & 389 & 2,17 \\
\hline 388 & 1 & 535 & 777 & 2,73 \\
\hline
\end{tabular}

Tabla VI

Autores más productivos.

\begin{tabular}{|l|c|}
\hline AUTOR & NÚM. TRABAJOS \\
\hline Gil, D. & 10 \\
\hline Furió, C. & 8 \\
\hline Moreira, M.A. & 7 \\
\hline Sanjosé, V. & 7 \\
\hline Banet, E. & 6 \\
\hline López Rupérez, F. & 6 \\
\hline
\end{tabular}

Figura 4

Evolución temporal de las citas recibidas por las referencias más citadas en Enseñanza de las Ciencias.

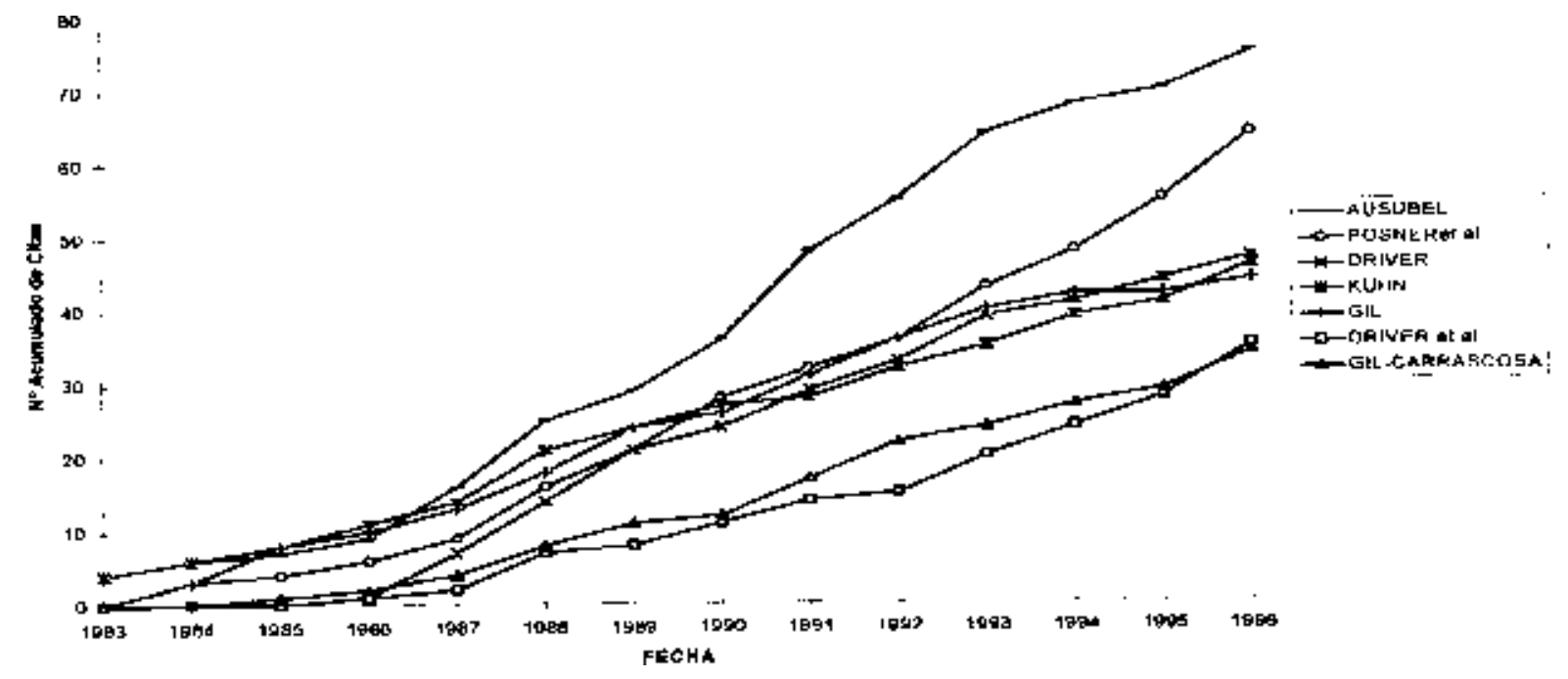

comprobado por el número de citas recibidas en general en el período de edición de la revista, a la vez que se observa una continuidad en los trabajos de Gil y de su escuela con una gran retroalimentación y una notable influencia de Posner y otros (1982) y Driver y otros (1986).

\section{CONCLUSIONES}

La revista Enseñanza de las Ciencias se ha tomado como soporte para el análisis de la evolución de la investigación en didáctica de las ciencias en nuestro país, ya que es, sin duda, la de mayor aceptación en nuestra comuni- 
Tabla VII

Relación de los autores más productivos y los trabajos más citados.

\begin{tabular}{|l|c|c|c|c|c|c|c|}
\hline & AUSUBEL & $\begin{array}{c}\text { POSNER } \\
\text { ET AL. } \\
(1982)\end{array}$ & DRIVER & KUHN & GIL & $\begin{array}{c}\text { DRIVER } \\
\text { ET AL. } \\
(1985)\end{array}$ & $\begin{array}{c}\text { CARRASCOSA } \\
\text { GIL }\end{array}$ \\
\hline GIL & 4 & 7 & 6 & 2 & 7 & 0 & 7 \\
\hline FURIÓ & 3 & 1 & 1 & 3 & 3 & 0 & 0 \\
\hline MOREIRA & 4 & 1 & 0 & 0 & 0 & 0 & 0 \\
\hline SANJOSÉ & 2 & 2 & 1 & 1 & 1 & 2 & 1 \\
\hline BANET & 0 & 1 & 3 & 0 & 0 & & 0 \\
\hline LÓPEZ & 0 & 1 & 0 & 1 & 0 & & 1 \\
\hline
\end{tabular}

Figura 5

Curva de Bradford de los autores más productivos.

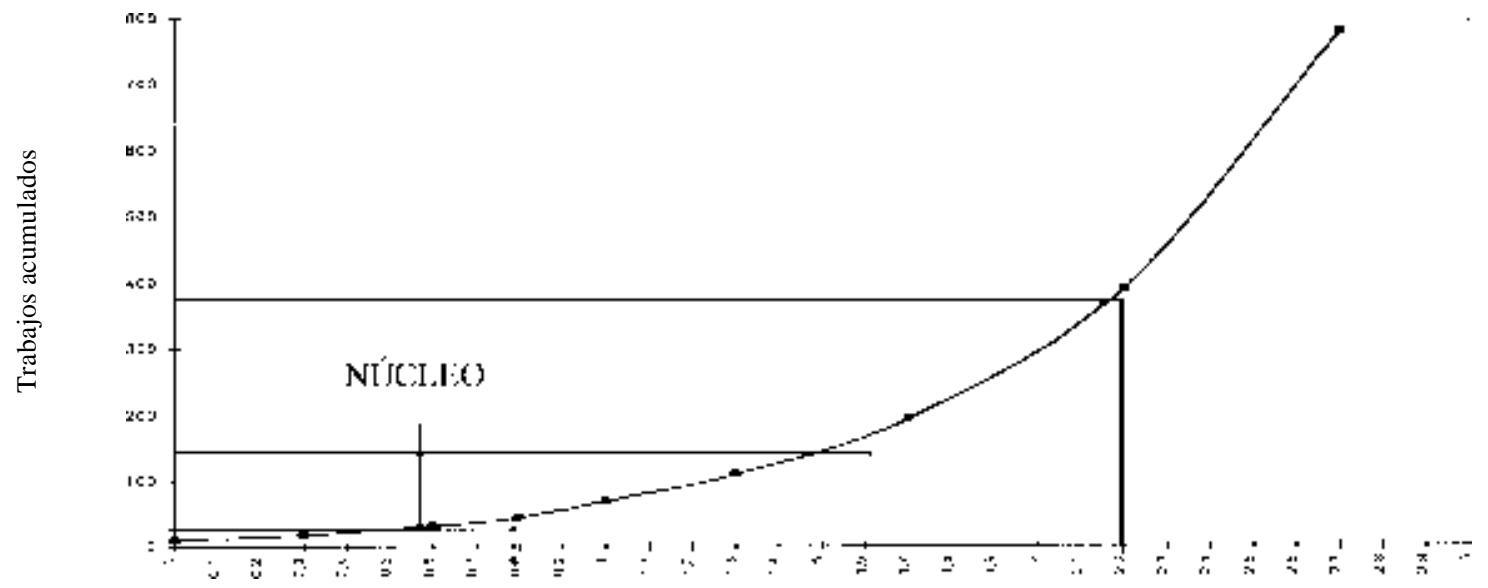

Log. autores acumulados

dad científica en lo referente a la enseñanza de las ciencias en niveles medios y universitarios.

Del estudio de las fuentes de información de los autores que publican en Enseñanza de las Ciencias, caben destacar las siguientes conclusiones:

1) El número de referencias citadas en los trabajos de la revista Enseñanza de las Ciencias se ha ido estabilizan- do a lo largo del período estudiado, siendo actualmente del orden de 30 referencias por trabajo, que parece adecuado para dar una base suficiente a los lectores interesados en el tema del artículo.

2) Durante los primeros años de publicación de la revista se citaba un mayor número de referencias de libros, y estas referencias han ido paulatinamente enfocándose hacia las revistas de didáctica, y en especial en los 
últimos años, hacia la propia revista Enseñanza de las Ciencias, denotando así la importancia que los propios autores han ido dando a las publicaciones de la misma.

3) Existe un núcleo de 7 referencias con más de 34 citas recibidas en trabajos publicados en Enseñanza de las Ciencias, en el que se pone de manifiesto el interés que las líneas de investigación relacionadas con el estudio de los esquemas conceptuales de los alumnos han tenido durante este período.

4) Sin embargo, una estabilización en algunas de las referencias más citadas en este campo parece indicar que cabría desear una renovación de las líneas de investigación en didáctica de las ciencias en España, ya que los preconceptos y errores previos de los alumnos han sido ya suficientemente estudiados y analizados durante los

\section{REFERENCIAS BIBLIOGRÁFICAS}

BRADFORD, S.C. (1948). Documentation. Crosby Lockwood: Londres.

GIL, D. (1994). Diez años de investigación en didáctica de las ciencias: realizaciones y perspectivas. Enseñanza de las Ciencias, 12, pp. 154-164.

LÓPEZ, J., SALVADOR, A. y DE LA GUARDIA, M. (1985). Estudio bibliométrico de la literatura científica sobre determinación de elementos metálicos en aceites lubricantes por espectroscopia de absorción atómica. Revista Española de Documentación Científica, 8(3), pp. 201-213. últimos años, y hay otros aspectos de la didáctica de las ciencias que precisarían una mayor atención en la actualidad.

5) Durante el período estudiado, hay seis autores que han publicado más de seis trabajos en la revista Enseñanza de las Ciencias, algunos de ellos vinculados a la propia revista, por lo que sería de interés una mayor apertura hacia docentes de todos los niveles de la enseñanza de las ciencias que ayudase a concienciar a los mismos de la necesidad de profundizar en la investigación didáctica en sus respectivos centros de trabajo.

En consecuencia, estas conclusiones se pueden extrapolar en general a los estudios en investigación y didáctica de las ciencias realizadas en España durante el período considerado.
LÓPEZ, J., LOSADA, J., SALVADOR, A. y DE LA GUARDIA, M. (1986). La evolución de la química analítica en España vista a través de un estudio estadístico y bibliométrico de la revista Química Analítica. Revista Española de Documentación científica, 9(4), pp. 337-349.

LÓPEZ CALAFÍ, J. (1995). El reconocimiento de la materia, un enfoque para la enseñanza de la química. Tesis doctoral. Universitat de València.

MOREIRA, M.A. (1994). Diez años de revista Enseñanza de las Ciencias: de una ilusión a una realidad. Enseñanza de las Ciencias, 12 , pp. 147-153.

[Artículo recibido en febrero de 1997 y aceptado en febrero de 1998.] 
ANEXO I

Referencias más citadas ordenadas según el número de citas recibidas.

Núm. de citas

75

64

46

Referencia citada

AUSUBEL, D.P., NOVAK, J.D. y HANESIAN, H. (1976). Psicología educativa: un punto de vista cognoscitivo. México: Trillas. Ed. orig., 1968, Educational Psychology: A cognitive view. Nueva York: Holt, Rinehart \& Winston.

POSNER, G.S., STRIKE, K.A., HEWSON, P.W. y GERTZOG, W.A. (1982). Acommodation of Scientific Conception: Towards a Theory of Conceptual Change. Science Education, 66, pp. 211-227.

DRIVER, R. (1986). Psicología cognoscitiva y esquemas conceptuales de los alumnos. Enseñanza de las Ciencias, 4 (1), p. 315.

KUHN, T.S. (1962). The structure of scientific revolutions. Chicago: University of Chicago Press. Trad. cast., 1971. La estructura de las revoluciones científicas. México: Fondo de Cultura Económica.

GIL PÉREZ, D. (1983). Tres paradigmas básicos en la enseñanza de las ciencias. Ensenanza de las Ciencias, Vol. 1(1), pp. 26-33.

DRIVER, R. , GUESNE, E. y TIBERGHIEN, A. (1985). Children's ideas in Science. Milton Keynes, Open University Press. Trad. cast., 1988. Ideas científicas en la infancia y la adolescencia. Madrid: Morata.

GIL PÉREZ, D. y CARRASCOSA, J. (1985). Science Learning as a conceptual and methodological change. European Journal of Science Education, 7(3), pp. 231-236.

NOVAK, J.D. y GOWIN, D.B. (1984). Learning how to learn. Nueva York: Cambridge University Press. Trad. cast., 1988. Aprendiendo a aprender. Barcelona: Martínez Roca.

SHAYER, M. y ADEY, P. (1981). Towards a Science of Science Teaching. Londres: Heinemann educational books. Trad. cast. La ciencia de enseñar ciencias. 1984. Madrid: Narcea.

CARRASCOSA, J. y GIL, D. (1985). La metodología de la superficialidad y el aprendizaje de las ciencias. Enseñanza de las Ciencias, 3(2), pp. 113-120.

NOVAK, J.D. (1977). A theory of education. Ithaca, N.J.: Cornell University Press. Trad. cast., 1982. Teoría y práctica de la educación. Madrid: Alianza Editorial.

GIL PÉREZ, D.,1986. La metodología científica y la enseñanza de las ciencias: unas relaciones controvertidas. Enseñanza de las Ciencias, 4(2), pp. 111-121.

DRIVER, R. y OLDHAM, V. (1986). A constructivist approach to curriculum development in science. Studies in Science Education, 13, pp. 105-122.

OSBORNE, R.J. y WITTROCK, M.C. (1983). Learning science: A generative process, Sci. Educ., 67(4), pp. 479-508.

INHELDER, B. y PIAGET, J. (1955). De la logique de l'enfant a la logique de l'adolescent. Essais sur la construction des structures opératoires formalles. París: PUF. Trad. cast. de M.T. Cevasco, 1972. De la lógica del niño a la lógica del adolescente. Buenos Aires: Paidós.

OSBORNE, R. y FREYBERG, P. (1985). Learning in Science. The implications of Children's science. Heinemann Publishers. Trad. cast., 1991, El aprendizaje de las ciencias. Implicaciones en la ciencia de los alumnos. Madrid: Narcea ediciones.

VIENNOT, L. (1979). Spontaneous reasoning in elementary dynamics. European Journal of Science Education, 1, pp. 205-221.

DRIVER, R. y ERICKSON, G. (1983). Theories in action: some theoretical and empirical issues in the study of conceptual frameworks in science. Studies in Science Education, 10, pp. 37-60.

HODSON, D. (1985). Philosophy of Science. Science and science Education. Studies in Science Education, Vol. 12, pp. 25-67.

POZO, J.L. (1987). Aprendizaje de la ciencia y pensamiento causal. Madrid: Editorial Visor.

BACHELARD, G. (1938). La formation de l'esperit scientifique. París: Vrin. Trad. cast., 1972. La formacion del espíritu científico. Buenos Aires. Siglo XXI.

DRIVER, R. (1988). Un enfoque constructivista para el desarrollo del currículo en ciencias. Enseñanza de las Ciencias, 6(2), pp. 109-120.

GIORDAN, A. (1982). La enseñanza de las ciencias. Madrid: Siglo XXI. Ed. orig., 1978. Une pédagogie pour les sciences expérimentales. París: Centurion. 
HEWSON, P.W. (1981). A conceptual change approach to learning science. European Journal of Science Education, Vol. 3(4), pp. 383-396.

TOULMIN, S. (1972). Human understanding - Vol 1. The collective usage and evolution of concepts. Princeton: Princeton University Press. Trad. cast., 1977. La comprensión humana, 1. El uso colectivo y la evolución de los conceptos. Madrid: Alianza Editorial.

GIL PÉREZ, D. (1993). Contribución de la historia y de la filosofía de las ciencias al desarrollo de un modelo de enseñanza-aprendizaje como investigación. Enseñanza de las Ciencias, 11(2), pp. 197-212.

NOVAK, J. D. (1987). Human Constructivism: Toward a Unity of Psychological and Epistemological Meaning Making, en Novak, J.D. (ed.). Proceedings of the Second International Seminar on Misconceptions and Educational Strategies in Science and Mathematics. Ithaca, Nueva York: Departamento de Educación, Universidad de Cornell. Versión cast. en Enseñanza de las Ciencias, 6, pp. 213-223.

GIL, D., CARRASCOSA, J., FURIÓ, C. y MARTÍNEZ-TORREGROSA, J. (1991). La enseñanza de las ciencias en la educación secundaria. Barcelona: ICE/Horsori.

VIENNOT, L. (1979). La raisonnement spontané en dynamique élémentaire. París: Hermann.

SALTIEL, E. y VIENNOT, L. (1985). ¿Qué aprendemos de las semejanzas entre las ideas históricas y el razonamiento espontáneo de los estudiantes? Enseñanza de las Ciencias, 3(2), pp. 137-144.

CLEMENT, J. (1982). Students' preconceptions in introductory mechanics. Am. J. Phys., 50, pp. 66-71.

HASHWEH, M.Z. (1986). Towards an explanation of conceptual change. European Journal of Science Education, 8(3), pp. 229-249. resolver problemas. México: Trillas.

GILBERT, J.K. y WATTS, D.M. (1983). Concepts, misconceptions and alternative conceptions: changing perspectives in science education. Studies in Sc. Education, 10, pp. 61-98.

HIERREZUELO, J. y MONTERO, A. (1989). La ciencia de los alumnos. Su utilización en la didáctica de la física y la química. Barcelona: Laia/MEC.

POPPER, K.R. (1959). La lógica de la investigación científica. Madrid: Tecnos, 1972.

SOLBES, J. y VILCHES, A. (1989). Interacciones CTS: un instrumento de cambio actitudinal. Enseñanza de las Ciencias, 7(1), pp. 14-20.

GAGNÉ, R.M. (1971). Las condiciones del aprendizaje. Madrid: Aguilar. Ed. orig., 1965, The conditions of learning. Nueva York: Holt, Rinehart \& Winston.

MATTHEWS, M.R. (1990). History, Philosophy and Science Teaching: A Rapprochement. Studies in Science Education, 18, pp. 25-51.

MILLAR, R. y DRIVER, R. (1987). Beyond processes. Studies in Science Education, 14, pp. 33-62.

McDERMOTT, L.C. (1984). Research on Conceptual Understanding in Mechanics. Physics Today, 37, pp. 24-32.

PIAGET, J. (1970). La epistemología genética. Barcelona: Redondo.

DRIVER, R. y EASLEY, J. (1978). Pupils and paradigms: a review of the literature related to concept development in adolescent science student. Studies in Science Education, 5, pp. 61-84.

GIL PÉREZ, D. y MARTÍNEZ TORREGROSA, J. (1983). A Model for problem solving in accordance with scientific methodology. European Journal in Science Education, Vol. 5, pp. 447-457.

GIORDAN, A. y DE VECCHI, G. (1987). Les origines du savoir. Des conceptions des apprenants aux concepts scientifiques. Neuchatel, París: Delachaux et Niestlé. Trad. cast., 1988. Los orígenes del saber. Sevilla: Díada.

HEWSON, P.W. y HEWSON, G.N. (1988). An Appropriate conception of teaching science: A view from studies of science learning. Science Education, Vol. 72(2), pp. 597-614.

OSBORNE, R. y WITTROCK, M. (1985). The Generative Learning Model and its Implications for Science Education. Studies in Science Education, Vol. 12, pp. 59-87.

GIL, D. (1985). El futuro de la enseñanza de las ciencias: algunas implicaciones de la investigación educativa. Revista de Educación, 278, pp. 27-38.

HODSON, D. (1988). Towards a philosophically more valid science curriculum. Science Education, 72(1), p. 1940.

KELLY, G.A. (1963). The Psychology of Personals Constructs. Nueva York: W.W. Norton. 
PIAGET, J. y GARCÍA, R. (1981). Psychogenèse et histoire des Sciences. París: PUF. Trad. cast., 1982. Psicogénesis e historia de la ciencia. México: Siglo XXI.

SOLÍS, R. (1984). Ideas intuitivas y aprendizaje de las ciencias. Enseñanza de las Ciencias, Vol. 2, pp. 8389.

T teaching. Journal of Research in Science Teaching, 26(2), pp. 105-120.

WATTS, D.M. y ZYLBERSZTASN, A. (1981). A survey of some children's ideas about force. Phis. Educ., 16(6), pp. 360-365.

YAGER, R.E. y PENICK, J.E. (1986). Perceptions of four age groups towards science classes, teachers and the value of science. Sci. Education, 70(4), pp. 355-364.

YAGER, R.E., y PENICH, J.E. (1983). Analysis of the Current Problems with School Science in the United Stated of America. European Journal of Science Education, Vol. 5, pp. 463-469.

AIKENHEAD, G.S. (1985). Collective decision making in the social context of science. Science Education, 69(4), pp. 453-475. Amplia reseña en Enseñanza de las Ciencias, 1986, 4(1), pp. 67-69.

FEYERABEND, P. (1987). Contra el método. Barcelona: Ariel.

GIL, D. y MARTÍNEZ-TORREGROSA, J. (1987). Los programas guía de actividades: una concreción del modelo constructivista de aprendizaje de las ciencias. Investigación en la Escuela, 3, pp. 3-12.

GILBERT, J.K., OSBORNE, R.J. y FENSHAM, P.J. (1982). Children's Science and its consequences for teaching. Science Education, 66, pp. 623-633.

GIORDAN, A. (1983). L'élève et/ou les connaisances scientifiques. París: Peter Lang.

LINN (1987). Establishing a Research Base for Science Education: Challenges, Trends and Recommendations. Journal of Research in Science Teaching, 24(3), pp. 191-216.

NUSBAUM, J. y NOVIK, S. (1982). Alternative frameworks conceptual conflict and accommodation: towards a principled teaching strategy. Instructional Science, 11, pp. 183-200.

OSBORNE, R.J., BELL, B.F. y GILBERT, J.K. (1983). Science teaching and children's views of the world. Eur. J. Sci. Educ., Vol. 5, pp. 114.

PIAGET, J. (1975). Introducción a la epistemología genética. Buenos Aires: Paidós.

ARANA, J., ESCUDERO, T., GARCÉS, R. y PALACIAN, E. (1987). Imagen de las asignaturas de ciencias en la transición de la educación básica a la secundaria. Enseñanza de las Ciencias, 5(1), pp. 10-15.

CARRASCOSA, J. y GIL, D. (1982). Los errores conceptuales en la enseñanza de la física. Un estudio de su persistencia. Primeras Jornadas de Investigación en Didáctica en Física y Química, ICE Valencia, pp. 277-281.

DEADMAN, J.A. y KELLY, P. (1978). What do secondary school boys understand about evolution and heredity before they are taught the topics? Journal of Biological Education, 1, pp. 7-15.

DRIVER, R., (1981). Pupil's alternative frameworks in science. European Journal or Science Education, Vol. 10, pp. 37-60.

FURIÓ, C. y HERNÁNDEZ, J. (1983). Ideas de los adolescentes de 11-15 años acerca de los gases. Enseñanza de las Ciencias, Vol. 1(1), pp. 83-91.

HOLTON, G. (1976). Introducción a los conceptos y teorías de las ciencias físicas, revisada y ampliada por S.G. Brush. Barcelona: Editorial Reverté.

LAWSON, A.L. (1978). The Development and Validation of Classroom Test of Formal Reasoning. Journal of Research in Science Teaching, Vol. 15, pp. 11-24.

NUSSBAUM, J. y NOVICK, S. (1981). Brainstorming in the clasroom to invent a model: a case study. School Science Review, 61, 221, pp. 771-779.

PENICK, J.E. y YAGER, R.E. (1986). Trends in science education: some observations of exemplary programmes in the United States. European Journal of Science Education, 8(1), pp. 18.

RESNICK, L.B. (1983). Mathematics and science learning: A new conception. Science, Vol. 220, pp. 477 478.

SERRANO, T. (1986). Representaciones de los alumnos en biología: estado de la cuestión y problemas de su investigación en el aula. IV Jornadas de Estudio sobre la Investigación en la Escuela, pp. 157-162. 


\section{ANEXO II}

Autores más productivos de la revista Enseñanza de las Ciencias ordenados según el número de trabajos.

\begin{tabular}{|c|c|c|c|}
\hline NÚM. TRAB. & AUTOR & NÚM. TRAB. & AUTOR \\
\hline 10 & Gil, D. & 3 & Novack, J.D. \\
\hline 8 & Furió, C. & 3 & Oliva, J.M. \\
\hline 7 & Moreira, M.A. & 3 & Oñobre, A. \\
\hline 7 & Sanjosé, V. & 3 & Otero, J. \\
\hline 6 & Banet, E. & 3 & Pacca, J.L.A. \\
\hline 6 & López Rupérez, F. & 3 & Perales, F.J. \\
\hline 5 & Barberá, O. & 3 & Pesa, M. \\
\hline 5 & Escudero, T. & 3 & Sáez, M.J. \\
\hline 5 & Jiménez, M.P. & 3 & Salinas, J. \\
\hline 5 & Núñez, F. & 3 & Sánchez, J.M. \\
\hline 5 & Palacios, C. & 3 & Solaz, J.J. \\
\hline 4 & Colombo, L. & 3 & Villani, A. \\
\hline 4 & Garrote, R. & 2 & Aliberas, J. \\
\hline 4 & Giordan, A. & 2 & Álvarez, J.A. \\
\hline 4 & Llopis, R. & 2 & Anguita, F. \\
\hline 4 & Mata, A. & 2 & Arana, J. \\
\hline 4 & Quílez, J. & 2 & Astolfi, J.P. \\
\hline 4 & Sebastiá, J.M. & 2 & Axt, R. \\
\hline 4 & Seminario & 2 & Bullejos, J. \\
\hline 4 & Solbes, J. & 2 & Caamaño, A. \\
\hline 4 & Vázquez, A. & 2 & Cachapuz, F. \\
\hline 3 & Acevedo, J.A. & 2 & Camacho, E. \\
\hline 3 & Aguirre, I. & 2 & Camps, J. \\
\hline 3 & Anta, C. & 2 & Cañal, P. \\
\hline 3 & Borsese, A. & 2 & Carrascosa, J. \\
\hline 3 & Brincones, I. & 2 & Casadella J. \\
\hline 3 & Brosseau, C. & 2 & Castro, E.A. \\
\hline 3 & Caballer, M.J. & 2 & Chastrette, M. \\
\hline 3 & De Posada, J.M. & 2 & Cudmani, C. \\
\hline 3 & Dibar, M.C. & 2 & De Jaime, M.C \\
\hline 3 & Gagliardi, R. & 2 & Díaz, F. \\
\hline 3 & Garret, R.M. & 2 & Díaz, J. \\
\hline 3 & Gutiérrez, R. & 2 & Domènech, A. \\
\hline 3 & Lang, F. & 2 & Driver, R. \\
\hline 3 & Llorens, J.A. & 2 & Espinosa, J. \\
\hline 3 & Lucas, A.M. & 2 & Favieres, A. \\
\hline 3 & Nieda, J. & 2 & Fernández Fern \\
\hline
\end{tabular}




\begin{tabular}{|c|c|c|c|}
\hline NÚM. TRAB. & AUTOR & NÚM. TRAB. & AUTOR \\
\hline 2 & Filloy, E. & 2 & Molina, E. \\
\hline 2 & Fontes, M.A. & 2 & Mondelo, M. \\
\hline 2 & Galache, M.I. & 2 & Monros, M.A. \\
\hline 2 & Galagovsky, L.R. & 2 & Navarro, V. \\
\hline 2 & Garcés, R. & 2 & Neto, A.J. \\
\hline 2 & García Barquero, $\mathrm{P}$. & 2 & Níaz, M. \\
\hline 2 & García Bayonas, A. & 2 & Núñez, J.M. \\
\hline 2 & García Hourcade, J.L. & 2 & Ortega, P. \\
\hline 2 & García, A.M. & 2 & Palacian, E. \\
\hline 2 & García, S. & 2 & París, R. \\
\hline 2 & Gascón Pérez, J. & 2 & Pastor, J. \\
\hline 2 & Giménez, I. & 2 & Pedrinaci, E. \\
\hline 2 & Goberna, M.A. & 2 & Perzlandazábal, M.C. \\
\hline 2 & Gómez Alfonso, B. & 2 & Pesoa, A.M. \\
\hline 2 & Gómez, M.A. & 2 & Pintó, R. \\
\hline 2 & Gutiérrez, F.A. & 2 & Porlán, R. \\
\hline 2 & Hernán, F. & 2 & Puig, L. \\
\hline 2 & Hewson, P.W. & 2 & Rodríguez Ávila, C. \\
\hline 2 & Hierrezuelo, J. & 2 & Rodríguez, A. \\
\hline 2 & Ianniello, M.G. & 2 & Rodríguez, L.M. \\
\hline 2 & Izquierdo, M. & 2 & Roman, $\mathrm{T}$. \\
\hline 2 & Jiménez, E. & 2 & Rosado, L. \\
\hline 2 & Joshua, S. & 2 & Saltiel, E. \\
\hline 2 & Kempa, R.F. & 2 & Saura, J.P. \\
\hline 2 & Labur, C.E. & 2 & Sevilla, C. \\
\hline 2 & Larcher, C. & 2 & Soler, J. \\
\hline 2 & Latorre, A. & 2 & Ten, A.E. \\
\hline 2 & Lillo, J. & 2 & Tiberghien, A. \\
\hline 2 & Llinares, S. & 2 & Valdés, R. \\
\hline 2 & López Cancio, J.A. & 2 & Varela, P. \\
\hline 2 & Manrique, M.J. & 2 & Vázquez, J. \\
\hline 2 & Marín, N. & 2 & Viard, J. \\
\hline 2 & Martinand, J.L. & 2 & Vicentini, M. \\
\hline 2 & Martínez, C. & 2 & Viennot, L. \\
\hline 2 & Martínez, J. & 2 & Vilches, A. \\
\hline 2 & Mathews, M.R. & 2 & Zalamea, E. \\
\hline 2 & Mellado, V. & & \\
\hline
\end{tabular}

